\title{
Konzo: a distinct disease entity with selective upper motor neuron damage
}

T Tylleskär, W P Howlett, H T Rwiza, S-M Aquilonius, E Stålberg, B Lindén, A Mandahl, H C Larsen, G R Brubaker, H Rosling

International Child

Health Unit,

Department of

Pediatrics, University

Hospital, Uppsala,

Sweden

T Tylleskär

H Rosling

Kilimanjaro Christian

Medical Center,

Moshi, Tanzania

W P Howlett

Department of

Internal Medicine,

Muhimbili Medical

Center, Dar-es-

Salaam, Tanzania

H T Rwiza

Department of

Neurology, University

Hospital, Uppsala,

Sweden

S-M Aquilonius

Department of

Clinical

Neurophysiology,

University Hospital,

Uppsala, Sweden

E Stålberg

Department of

Radiology, Falun

Hospital, Falun,

Sweden

B Lindén

Department of

Ophthalmology,

University Hospital,

Uppsala, Sweden

A Mandahl

Department of

Audiology, University

Hospital, Uppsala,

Sweden

H C Larsen

Shirati Hospital,

Musoma, Mara

Region, Tanzania

G R Brubaker

Correspondence to:

Dr T Tylleskär,

International Child Health

Unit (ICH), Department of

Pediatrics, University

Hospital, S-751 85 Uppsala, Sweden.

Received 18 May 1992

and in revised form

16 October 1992.

Accepted 28 October 1992

\begin{abstract}
Two Tanzanian patients with konzo were severely disabled by a non-progressive spastic paraparesis, since the sudden onset during an epidemic six years earlier. At the time of onset they had a high dietary intake of cyanide from exclusive consumption of insufficiently processed bitter cassava roots. MRI of brain and spinal cord were normal but motor evoked potentials on magnetic brain stimulation were absent, even in the only slightly affected upper limbs. Other neurophysiological investigations were largely normal but the more affected patient had central visual field defects. Konzo is a distinct disease entity with selective type upper motor neuron damage.
\end{abstract}

\section{$(\mathcal{F}$ Neurol Neurosurg Psychiatry 1993;56:638-643)}

Konzo is an upper motor neuron disease, characterised by abrupt onset of a varying degree of symmetrical, isolated, and permanent but non-progressive spastic paraparesis. It was first described in Zaire in 1938 and is named after the local designation in the first report. ${ }^{1}$ In the last decade it has been reported from remote rural areas of Mozambique, Tanzania, and Zaire. The uniform epidemiological and clinical findings have identified konzo as a distinct disease entity induced by a combined effect of high cyanide and low sulphur intake from exclusive consumption of insufficiently processed bitter cassava roots. ${ }^{2}$ The study of konzo has formerly been limited to clinical examinations during field surveys.

\section{Subjects and methods}

Two male konzo patients aged 25 and 19 years were invited to Sweden in October 1991. They were diagnosed in 1985 during an epidemic in Tarime district, ${ }^{13}$ situated east of Lake Victoria in the northern part of Tanzania.

The study was approved by the ministry of health of Tanzania and the ethical committee of Uppsala University. Local civil and health authorities informed the patients and their families about the aim and procedures to be undertaken and obtained written consent from the patients to participate. A local health worker accompanied the patients to Sweden and acted as interpreter.
Histories were taken and neurological examinations were carried out on both patients during visits in their homes in May 1985 and repeated in 1986, 1988 (WPH), and again in Sweden in 1991 (S-M A).

Magnetic resonance imaging (spin echo) of the brain and spinal cord was performed with a Philips T5 MR-scanner ( 0.5 Tesla unit) without use of intravenous contrast. $\mathrm{T} 1$ weighted, proton density, and T2 weighted images were obtained in sagittal, coronal, and axial planes of the brain and in the sagittal plane of the whole spinal cord. Axial proton density and T2 weighted images over the conus medullaris were also obtained.

Neurophysiological investigations were performed with conventional techniques including motor and sensory nerve conduction, small fibre tests such as respiratory dependent heart rate variation, ${ }^{4}$ thermal perception thresholds for warmth and cold and pain threshold for heat and cold; concentric electromyography (EMG) both at rest, at slight voluntary contraction and during maximal contraction (automatic turn/amplitude analysis, ${ }^{5}$ single fibre EMG, fibre density measurement, jitter analysis, blink reflexes, electroenchephalography (EEG), somatosensory evoked potentials (SEP), full field visual evoked potentials (VEP), and brainstem evoked response audiometry (BERA). Transcranial stimulation of the motor cortex was performed with a magnetic stimulator to elicit motor evoked potentials (MEP). Stimulation was also performed over the C7 vertebra. Pure tone audiometry and caloric stimulation of the vestibularis were studied in conventional ways. Electronystagmography (ENG) in a dark room supervised by an infrared camera was used for the registration of spontaneous nystagmus, head shake nystagmus, positional nystagmus, and gaze nystagmus at $30^{\circ}$ eye deviation. Smooth pursuit movements of $60^{\circ}$ with a fixed velocity of $20 \% 10$ movement to the right and 10 to the left was recorded with ENG and analysed by computer for velocity, accuracy, and superimposed saccades according to standardised procedure. ${ }^{6}$ Voluntary saccades (that is, rapid eye movements) of $60^{\circ}$ was performed 20 times to the right and 20 times to the left analysed by computer for start latency, accuracy, and velocity. ${ }^{6}$

Conventional ophthalmological investigations included: visual acuity, eye pressure, direct/consensual pupillary reflex, corneal sensitivity, colour vision, dark adaptation, and binocularity. Visual fields were examined 
with Goldmann perimetry. The ocular fundi were photographed and scrutinised for defects in the nerve fibre layer.

Serum thiocyanate was used as biomarker for dietary cyanide exposure. ${ }^{2}$ An extensive battery of haematological and biochemical routine analyses were carried out including analyses of blood cells, platelets, haemoglobin, serum levels of electrolytes, different proteins, enzymes, vitamin B6, B12, thyroid hormones, a plasma electrophoresis, and routine urine tests. Serological testing included: IgM anti-hepatitis A virus, hepatitis B surface antigen, anti-hepatitis $C$ virus, antibodies to syphilis, retroviruses HIV-1 +2 (Enzygnost AntiHIV1 + 2 EIA, Wellcosyme Recombinant HIV-1 EIA, PCR HIV-1), HTLV-I (Abbot HTLV-I EIA, PCR HTLV-I). Characterisation of lymphocyte subpopulations by immunofluorescence and flow cytometry as well as an attempt to isolate HIV-1 and HTLV-I from blood specimens was performed. Direct microscopy of faeces was performed for cysts and eggs of intestinal parasites and direct microscopy of blood films for malaria parasites, trypanosomes, and filaria. ELISA serology for amoeba, echinococcus, filaria, schistosoma, and immunofluorescence for leishmania, trypanosomes, giardia, and malaria was performed. The cerebrospinal fluid was screened for syphilis and examined for cells, protein, glucose, and lactate. A protein electrophoresis and an isoelectric focusing was done.

\section{Results}

PATIENT 1

This man was born in 1966, the eldest of three children. There was no family history of neurological disorder. He was healthy until the 16 March 1985. The night before the onset of his illness he attended a local dance and experienced no difficulties with his legs. Later that night at home on the way to the latrine he noticed a weakness of the legs. The following morning the weakness was more pronounced and the legs were trembling on standing. He also experienced some sensations in the legs, and felt "as if the skin was not his". However, he managed to go and work in the field and when returning for lunch he sat down on a chair to rest. Since then he has never been able to walk again. There was a further deterioration during the first days with weakness in the arms and slurred speech, but no visual or hearing impairments. He was confined to bed for approximately two months after which speech difficulties cleared and there was a functional improvement in his legs and especially in his arms allowing him to stand with the support of one person. He did not have any bladder problems. About one year later, he had an episode of visual impairment when he was unable to read a book but this cleared in some weeks. In 1989 he married and the couple have since then had two children. His disability has remained unchanged apart from the functional improvements in the first months. He is able to move around with a four point walking frame and he makes his living as a shoe maker.

In the month preceding the onset the family was almost exclusively eating cassava, the only crop to survive the drought in 1985 . His youngest brother, born in 1973, was similarly but less severely affected in March 1985.

\section{PATIENT 2}

This man was born in 1972 as the fifth born in a family of 12 children. There was no family history of neurological disorder. He was healthy until March 1985 when one night he noticed weakness in both legs while walking to the latrine. He returned to bed with difficulty but in the morning he was unable to stand, and over the next three days the weakness increased and he was unable to get out of bed. At this stage he also experienced difficulties in speaking, visual disturbances and some tingling sensations in his legs, and a cold feeling in his feet. He also experienced clumsiness in his hands. He improved over some weeks and since May 1985 he is able to stand and to walk with the help of two sticks. His hoarseness cleared and his vision improved.

At the time of onset of the paralysis the diet of the family consisted almost exclusively of cassava because of drought. Out of the total household of two parents and eight living children four other siblings aged $6,11,12$ and 14 were similarly affected in March-April 1985. The only three to escape the disease were the oldest child who was away at a boarding school, the youngest who was still breast-feeding, and a four year old boy.

\section{CLINICAL FINDINGS *}

Figure 1 shows the two patients in October 1991, six years after onset. The clinical findings from the initial examination in May 1985, six weeks after the onset, and the examination in October 1991 were almost identical. Both patients were unable to walk unaided because of severe spastic paraparesis. The degree of disability on the expanded disability status scale (EDSS) was rated at $7 \cdot 0$ for patient 1 and 6.5 for patient 2 . This disability was characterised by extensive motor envolvement in the legs and a typical toe scissors gait on walking. The main abnormal neurological findings were in the legs in both patients and were as follows: hypertonia, most obvious in the strongest muscle groups-that is, hip adductors, knee extensors and ankle plantar flexors, bilateral sustained ankle clonus, loss of power (MRC range $0 / 5$ ankle dorsi flexors in patient 1 due to contractures, to $4 / 5$ knee flexors in the same patient and $3 / 5$ hip flexors to $4 \cdot 5 / 5$ plantar flexors in patient 2), hyperreflexia,

*A 7 minute video tape showing the main neurological findings and the magnetic stimulation is available from the author. ings and the magnetic stimulation is available from the author It is free of charge for colleagues from developing countries and for others we charge the equivalent of $£ 20$ sterling for production and postage. Unless otherwise requested we will distribute a VHS/PAL tape. 


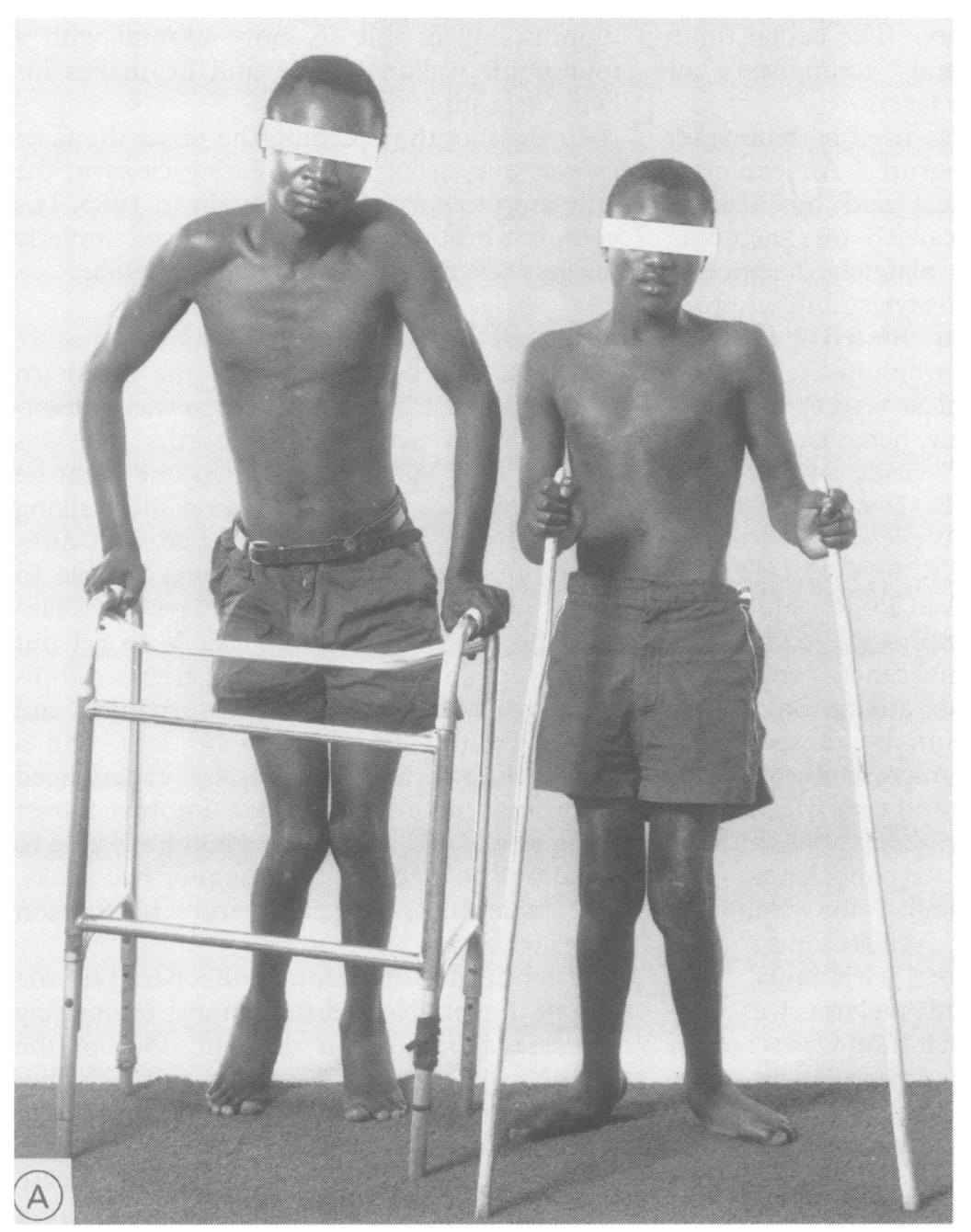

crossed adductor reflexes, and extensor plantar responses. Sensation (superficial and deep) and superficial reflexes were intact. In the upper limbs there was an obvious impairment of fine repetitive and rapid alternating hand movements affecting both patients. Patient 2 had a slight (MRC 4.5/5) loss of power affecting the extensors at the right elbow. Otherwise the upper limbs were normal. The cranial nerves and mental function were normal apart from an eye motion abnormality and a visual field defect (both presented below). The jaw jerk was absent. The rest of the neurological assessment including cerebellar, basal ganglia and autonomic function was normal.

\section{MRI AND NEUROPHYSIOLOGY}

On MRI no abnormalities were found in the motor cortex, the pyramidal tracts or elsewhere in the brain, brainstem, or the spinal cord.

The main neurophysiological finding was the complete absence of response at repeated magnetic stimulation of the motor cortex using maximal output of the stimulator at muscle rest and at various degrees of voluntary muscle contraction for facilitation; both in the affected legs and in the only slightly affected arms. There was twitching of facial muscles on magnetic stimulation and normal responses were obtained from arms upon

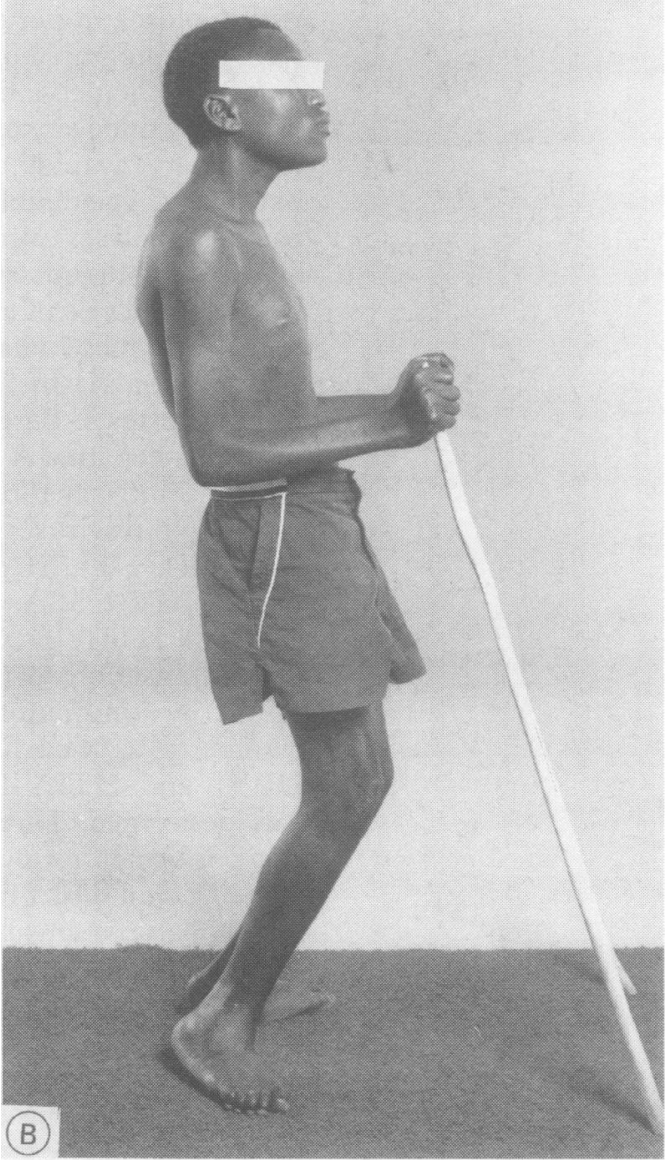

Figure (A) Frontal view of the patients showing the typical toe scissors gait and their usual walking aids. typical toe scissors gait and
$(B)$ Side view of patient 2.

stimulation over the neck at C7 level. Both patients showed normal peripheral nerve conduction, EMG, BERA, and blink reflexes. Respiratory dependent heart rate was also normal. In patient 1 the EEG amplitudes of all frequencies were abnormally low. The recordings showed nearly no $\alpha$ activity during wakefulness, drowsiness, hyperventilation, or photic stimulation. In patient 2 EEG was normal but sensory nerve action potentials were abnormally low and thermal thresholds were abnormally high. VEP showed asymmetry with longer latency from the right eye but the absolute values for each eye was within normal limits.

The pure tone audiometry, electronystagmography, and caloric reactions were normal. In the oculomotor test none of the patients had any eye muscle paralysis but both patients had pathological smooth pursuit movements with regard to velocity and by superimposed saccades. The voluntary saccades were normal in accuracy and velocity but the start latency was too slow (288-308 ms) in both patients.

\section{OPHTHALMOLOGY}

Patient 1 had bilateral central visual field defects and a corresponding atrophy of the papillomacular nerve fibre layer with temporal pallor of the optic discs. His colour vision (blue-yellow discrimination) was borderline. 
Patient 2 had small white spots in the macula lutea, compatible with a chloroquine retinopathy, and a vasculitis with arteriolar sheathing in the nasal part of the left retina. Other ophthalmological examinations were normal.

\section{LABORATORY INVESTIGATIONS}

Serum thiocyanate was $532 \mu \mathrm{mol} / 1$ for patient 1 and $230 \mu \mathrm{mol} / 1$ for patient 2 in May 1985 and 62 and $10 \mu \mathrm{mol} / 1$ respectively in October 1991 (reference value 8-76 $\mu \mathrm{mol} / \mathrm{l}$ ).

Other laboratory findings were normal except in patient 1 (reference values in parenthesis): white blood cell count 3.2 (4-9 10\%/1) with $26 \%$ polymorphonuclear cells; albumin 41 (42-55 g/l); $\alpha$-amylase 8.0 (1.4-5 $\mu \mathrm{kat} / \mathrm{l})$; plasma Ig G $21 \cdot 2(7 \cdot 0-18 \cdot 0 \mathrm{~g} / \mathrm{l})$, plasma IgM $3.62(0.4-2.75 \mathrm{~g} / \mathrm{l})$ but no monoclonal bands were found. Patient 2 had normal values except: albumin $36 \quad(42-55 \mathrm{~g} / \mathrm{l})$; $\alpha$-amylase $11.2(1.4-5 \mu \mathrm{kat} / \mathrm{l})$; alkaline phosphatase 9.4 (0.8-4.8 $\mu \mathrm{kat} / \mathrm{l})$; AST $0.92(<0.6$ $\mu \mathrm{kat} / \mathrm{l}) ;$ ALT 0.66 (<0.6 $\mu \mathrm{kat} / \mathrm{l}) ; \mathrm{LDH} 8.5$ (3.8-6.7 $\mu \mathrm{kat} / \mathrm{l})$, and plasma IgM 3.67 $(0 \cdot 4-2 \cdot 75 \mathrm{~g} / \mathrm{l})$.

Both patients were seronegative to HTLV1, HIV-1, HIV-2, syphilis, and hepatitis in 1985 as well as in 1991. No retroviruses could be isolated from the blood and CD4 +/CD8 + lymphocyte ratios were normal.

Microscopy of faeces and blood films were negative in both patients. ELISA serologies were negative for amoeba and echinococcus and weakly positive to filaria and schistosoma in both patients. Immunofluorescence for leishmania, giardia, and trypanosomes was weakly positive in both patients but strongly positive for malaria (1:5120 and 1:81920).

Patient 1 had a traumatic bleed on lumbar puncture and the collected CSF contained $7: 2 \times 10^{9} / 1$ erythrocytes, $24 \times 10^{6} / 1$ polymorphonuclear cells, and $18 \times 10^{6} / 1$ lymphocytes. The bleeding also increased the protein content to $700 \mathrm{mg} / 1$ (150-500 mg/l). CSF albumin was $198 \mathrm{mg} / 1(<290 \mathrm{mg} / \mathrm{l})$, and $\mathrm{IgG}$ $122 \mathrm{mg} / \mathrm{l}(<33 \mathrm{mg} / \mathrm{l})$. Plasma albumin was 37 $\mathrm{g} / 1$ (35-46 g/l) and IgG $21 \cdot 2 \mathrm{~g} / 1(7-15 \mathrm{~g} / \mathrm{l})$. The albumin ratio (CSF/plasma) was $5 \cdot 3$ $(<6.5)$ and IgG index $1 \cdot 0(<0 \cdot 7)$. Isoelectric focusing showed no oligoclonal bands. Glucose in CSF/blood was $3 \cdot 2 / 5 \cdot 7 \mathrm{mmol} / 1$ and CSF lactate was $1.4 \mathrm{mmol} / 1$. Syphilis test was negative.

Patient 2 had normal cerebrospinal fluid with no erythrocytes or polymorphonuclear cells and $1 \times 10^{6} / 1$ lymphocytes. The protein content was $240 \mathrm{mg} / \mathrm{l}$, albumin $98 \mathrm{mg} / \mathrm{l}$, and IgG $30.5 \mathrm{mg} / \mathrm{l}$. Plasma albumin was $36 \mathrm{~g} / \mathrm{l}$ and IgG $17 \cdot 7 \mathrm{~g} / 1(7-15 \mathrm{~g} / \mathrm{l})$ and the albumin ratio (CSF/plasma) was $2 \cdot 7$ and IgG index $0 \cdot 65$. Isoelectric focusing showed no oligoclonal bands. Glucose in CSF/blood was $3.9 / 5.7 \mathrm{mmol} / 1$ and CSF lactate was 1.9 $\mathrm{mmol} / 1$. Syphilis test was negative.

\section{Discussion}

The clinical findings in both patients were typical for konzo, that is, a sudden onset of a symmetric, isolated, and non-progressive spastic paraparesis, unchanged over time. The characteristic toe scissors gait, the symmetrically exaggerated and clonic reflexes in the lower limbs and the extensor plantar responses in the absence of sensory or autonomic disturbances are all constant findings in patients with severe konzo. ${ }^{17}$ In the villages of the two patients, konzo constitute two thirds of all locomotor disabilities ${ }^{3}$ and the patients studied belong to the most severely affected subjects.

A retrovirus aetiology of konzo has been suspected ${ }^{7}$ but the two konzo patients studied were seronegative to all retroviruses (HTLV1, HIV-1, and HIV-2), like all other konzo patients tested. ${ }^{127}$ Further tests such as PCR, virus isolation, and lymphocyte subpopulations showed no sign of retrovirus affection in the two patients. The distribution of HTLV-I associated myelopathy/tropical spastic paraparesis (HAM/TSP) and konzo overlap geographically ${ }^{8}$ but apart from negative serology konzo also differs clinically from HAM/TSP by its abrupt onset and non-progressive course. Plasma immunoglobulins were increased in both patients, as expected in subjects from an environment with high rate of infectious diseases. Patient 1 had a somewhat increased IgG index of CSF but no oligoclonal bands could be detected. Such elevated IgG index are occasionally found in non-inflammatory neurological diseases as a non-specific sign of neurodamage. ${ }^{9}$ An earlier study in Zaire also reported absence of oligoclonal bands in the CSF of konzo patients. ${ }^{7}$ In conclusion, neither this nor earlier epidemiological or clinical studies support the involvement of an infectious agent in konzo.

At the time of the onset in 1985 both patients subsisted on cassava that, because of food shortage, was insufficiently processed. ${ }^{13}$ They had high dietary cyanide intakes as supported by very high serum thiocyanate. High cyanide exposure has been verified at onset in all areas where konzo have been reported. This and other findings strongly indicate a toxiconutritional aetiology in konzo. ${ }^{12}$

Lathyrism is another toxiconutritional disease that is similar to konzo but their geographical distributions do not overlap and they are attributed to different types of monotonous diets. The only clinical differences between the diseases are the occasional visual involvement in severe konzo and the occasional autonomic involvement in lathyrism. ${ }^{10} \mathrm{~A}$ common final pathogenetic mechanism has been suggested ${ }^{1}$ but no comparison of the neurodamage in the two diseases can be made since similar neuroradiological and neurophysiological examinations are lacking for lathyrism.

The first interesting result of the present study is the absence of pathological findings on MRI in the two severely handicapped konzo patients. This is in sharp contrast to the extensive lesions found on MRI in patients with multiple sclerosis ${ }^{11}$ (MS) with the same degree of disability-that is, 
EDSS $\geqslant 6$. The multifocal lesions frequently seen on MRI in HAM/TSP ${ }^{12}$ differ in distribution and are less impressive than those found in patients with MS with a similar degree of disability. But in contrast to our normal findings in the two konzo patients, pathological MRI changes were found in 13 out of $14 \mathrm{HAM} / \mathrm{TSP}^{13}$ patients with a disability of EDSS $\geqslant 6$. Patients with motor neuron disease $^{14}$ have limited lesions along the pyramidal tract that are related to the severity of the disease. The normal MRI in konzo thus indicates a more selective pathogenetic mechanism than in MS, HAM/TSP, and motor neuron disease.

The second interesting result is the absence of motor evoked potentials on transcranial magnetic stimulation of the motor cortex in both konzo patients. Difficulties in exciting the motor cortex electrically or by magnetic stimulation have been seen in patients with hyperreflexia, spasticity ${ }^{15}$ and extensor plantar responses ${ }^{16}$ in relation to the severity of the upper motor neuron involvement. ${ }^{17}$ It is therefore not surprising that no response could be elicited in the severely affected legs but the inexcitability of the upper limbs is surprising since both patients had a good voluntary control of their arms. Only one study reports some few patients with motor neuron disease having absent responses after electrical brain stimulation in muscles with preserved voluntary control. ${ }^{18}$ Antiepileptic drug medications have also been reported to induce a dissociation between magnetic excitability and voluntary control ${ }^{19}$ although the patients in this study were not taking any medication.

Frequent involvement of the upper limbs manifested by weakness and hyperreflexia has been reported at onset of konzo. ${ }^{1}$ This usually clears but may persist in the more severely affected cases. We have observed a similar pattern in minimally affected lower limbs where residual disease is manifested by persistent hyperreflexia only. In the two patients studied the inability to make fine repetitive and alternating finger and hand movements in the absence of cerebellar disease is the only clinically detectable sign of minimal damage of the corticospinal tracts controlling the upper limbs. The complete inexitability of the arms to cortical stimulation, while supporting this clinical finding, also suggests that konzo may constitute a rare pattern of corticospinal failure where a subpopulation of neurons may be damaged at cortical or corticospinal level while relatively normal muscle power and function may be still preserved in the affected limbs. This supports the case for a further spectrum of minimally detectable or subclinical disease among the konzo affected populations. The abnormal findings in eye motion that is, saccadic eye movements and inability to rapidly alternate the fixation, are most easily interpreted as a bulbar analogue to the findings in the upper limbs.

The more severely affected patient in this study had central visual field defects and a corresponding atrophy of the papillomacular nerve fibre layer with temporal disc pallor. These findings are characteristic of a "toxic/deficiency optic neuropathy". ${ }^{20}$ Earlier studies of konzo have noted reduced visual acuity in severe cases of konzo, especially in the first weeks following onset. ${ }^{17}$ Both the optic neuropathy found in this study and earlier reported findings of reduced visual acuity show that in severe cases of konzo an optic nerve lesion may add to the isolated damage of corticospinal tracts.

\section{CYANIDE AND THE NERVOUS SYSTEM}

The types of neurodamage that has been attributed to cyanide seem to depend on the dose rate. Firstly it is well known that very high exposure leads to rapid death through an effect on central neurons. Secondly, the fact that sublethal acute intoxications may result in Parkinsonian symptoms following damage to basal ganglia has been shown on MRI in such patients. ${ }^{21}$ Thirdly, several years to decades of low dietary cyanide exposure from cassava has in Nigeria, been associated with a syndrome of slowly progressive ataxia, peripheral neuropathy, and optic atrophy. This syndrome is known as tropical ataxic neuropathy ${ }^{22}$ and is clinically distinct from konzo.

The abrupt corticospinal damage in konzo has been attributed to a fourth pattern of cyanide exposure, that is, several weeks of uninterrupted high but sublethal blood cyanide levels. ${ }^{2}$ These result from a high dietary cyanide intake in combination with impaired cyanide to thiocyanate conversion due to low sulphur intake. This metabolic pattern results from exclusive consumption of insufficiently processed bitter cassava roots without protein rich supplementary foods. The dietary situation has been identically extreme in all five areas of Africa where konzo has been well documented. ${ }^{12}$ From the present study we hypothesise that several weeks of high blood cyanide levels at a certain threshold, directly or after further metabolic events, cause an irreversible damage to a hitherto unidentified structure of some of the upper motor neurons.

The effect on CNS of the pattern of cyanide exposure associated with konzo has not been tested in an animal model. An observation of hindlimb paralysis in rats exposed to the combination of a high cyanide and low sulphur, while testing its diabetogenic effect, is so far the only experimental support for a cyanide aetiology of konzo. ${ }^{23}$ However, continuous exposure of primates to the related substance cyanate (OCN) resulted after six weeks in an abrupt onset of a permanent spastic paraparesis identical to konzo. ${ }^{24}$ As only minor histological changes were found in the corticospinal tracts of the cyanate exposed primates the toxicological mechanism may be the same as in konzo. The proposed toxiconutritional aetiology of konzo should be possible to confirm in an animal model. The inexcitability of motor cortex on transcranial stimulation may be used to identify subclinical forms of konzo in such 
models, as well as in future epidemiological studies.

The study would not have been possible without support from the Ministry of Health in Tanzania, Dr Festo Kavishe and Mr Nicolas Mlingi at the Tanzania Food and Nutrition Centre, the regional and local health authorities, Mr Daniel Kumbi, Miss Helena Karlén, and involved staff in different departments at Uppsala University and Falun hospital.

We also thank Professors Kjell Bergström and Anders Hemmingsson, Department of Diagnostic Radiology, and Professor Lars Klareskog, Department of Clinical Immunology and Transfusion Medicine, University Hospital, Immunology and Transfusion Medicine, University Hospital, Uppsala, Sweden for valuable advice and Dr Vasilios Kostulas, Department of Neurology, Huddinge Hospital, Stockholm for performing the isoelectric focusing on the cere-

The study was supported by SAREC, the Swedish Agency for Research Cooperation with Developing Countries.

1 Howlett WP, Brubaker GR, Mlingi N, Rosling H. Konzo, an epidemic upper motor neuron disease studied in an epidemic upper motor neuro

2 Tylleskär T, Banea $M$, Bikangi $N$, et al. Cassava cyanogens and konzo, an upper motoneuron disease cyanogens and konzo, an upper moto

3 Howlett W, Brubaker G, Mlingi N, Rosling H. A geographical cluster of konzo in Tanzania. $\mathcal{F}$ Trop Geograph Neurol 1992;2:102-8.

4 Stålberg E, Nogues $M$. Automatic analysis of heart rate variation, Part I: Method and reference values in healthy controls. Muscle Nerve 1989;12:993-1000.

5 Stålberg E, Chu J, Bril V, et al. Automatic analysis of the EMG interference pattern. Electroencephalogr Clin Neurophysiol 1983;56:672-81.

6 Bergenius J. Computerized analysis of voluntary eye movements. A clinical method for evaluation of smooth pursuit and saccades in oto-neurological diagnosis. Acta Otolaryngol (Stockh) 1984;98:490-500.

7 Carton H, Kazadi K, Kabeya, et al. Epidemic spastic paraparesis in Bandundu (Zaire). $f$ Neurol Neurosurg paresis in Bandundu

8 Kayembe K, Goubau P, Desmyter J, et al. A cluster of HTLV-1 associated tropical spastic paraparesis in Equateur (Zaire): ethnic and familial distribution. $\mathcal{f}$ Neurol Neurosurg Psychiatry 1990;53:4-10.

9 Link H, Tibbling G. Principles of albumin and IgG analyses in neurological disorders. II. Relation of the concentration of the proteins in serum and cerebrospinal fluid. Scand 7 Clin Lab Invest 1977;37:391-6.

10 Ludolph AC, Hugon J, Dwivedi MP, et al. Studies on the aetiology and pathogenesis of motor neuron diseases. 1 . Lathyrism: Clinical findings in established cases. Brain 1987;110:149-65

11 Truyen L, Gheuens J, Parizel P, et al. Long term followup of multiple sclerosis by standardized, non-contrast enhanced magnetic resonance imaging. $\mathcal{f}$ Neurol Sci 1991;106:35-40.

12 Newton M, Cruickshank K, Miller D, et al. Antibody to human T-lymphotropic virus type 1 in West-Indianborn UK residents with spastic paraparesis. Lancet 1987;i:415-6.

13 Kira J, Fujihara K, Itoyama Y, et al. Leucoencephalopathy in HTLV-I-associated myelopathy/tropical spastic paraparesis: MRI analysis and a two year follow-up study paresis: MRI analysis and a two year follow-up study

14 Sales Luís M, Hormigo A, Maurício C, et al. Magnetic resonance imaging in motor neuron disease. $\mathcal{f}$ Neurol $1990 ; 237: 471-4$.

15 Caramia M, Cicinelli P, Paradiso C, et al. Excitability changes of muscular responses to magnetic brain stimulation in patients with central motor disorders. Electroencephalogr Clin Neurophysiol 1991;81:243-50.

16 Ugawa Y, Shimpo T, Mannen T. Central motor conduction in cerebrovascular disease and motor neuron disease. Acta Neurol Scand 1988;78:297-306.

17 Hugon J, Lubeau M, Tabaraud F, et al. Central motor conduction in motor neuron disease. Ann Neurol 1987;22:544-6.

18 Thompson P, Day B, Rothwell J, et al. The interpretation of electromyographic responses to electrical stimulation of the motor cortex in diseases of the upper motor neuone. I Neurol Sci 1987;80:91-110.

19 Hufnagel A, Elger C, Marx W, Ising A. Magnetic motorevoked potentials in epilepsy: effects of the disease and of anticonvulsant medication. Ann Neurol 1990;28: 680-6.

20 Miller N, ed. Walsh and Hoyt's clinical neuroophthalmology. 4th ed. Baltimore/London: Williams \& Wilkins, 1982: vol I).

21 Carella F, Grassi M, Savoiardo M, et al. DystonicParkinsonian syndrome after cyanide poisoning: clinical and MRI findings. $\mathcal{F}$ Neurol Neurosurg Psychiatry 1988; 51:1345-8.

22 Osuntokun BO. Cassava diet, chronic cyanide intoxication and neuropathy in Nigerian Africans. World Rev Nutr Diet 1981;36:141-73.

23 McMillan DE, Geevarghese PJ. Dietary cyanide and tropical malnutrition diabetes. Diabetes Care 1979;2:202-8.

24 Shaw CM, Papayannopoulou T, Stamatoyannopuolos G Neuropathology of cyanate toxicity in Rhesus monkeys. Pharmacology 1974;12:166-76. 